\title{
Germinal center exclusion of autoreactive $B$ cells is defective in human systemic lupus erythematosus
}

\author{
Amedeo Cappione III,1 Jennifer H. Anolik, ${ }^{1}$ Aimee Pugh-Bernard,, Jennifer Barnard, ${ }^{1}$ \\ Paul Dutcher, ${ }^{1}$ Gregg Silverman, ${ }^{2}$ and Iñaki Sanz ${ }^{1}$ \\ ${ }^{1}$ Department of Medicine, University of Rochester School of Medicine, Rochester, New York, USA. ${ }^{2}$ UCSD, La Jolla, California, USA.
}

\begin{abstract}
Breach of $B$ cell tolerance is central to the pathogenesis of systemic lupus erythematosus (SLE). However, how $B$ cell tolerance is subverted in human SLE is poorly understood due to difficulties in identifying relevant autoreactive $B$ cells and in obtaining lymphoid tissue. We have circumvented these limitations by using tonsil biopsies to study autoreactive B cells (9G4 B cells), whose regulation is abnormal in SLE. Here we show that 9G4 $B$ cells are physiologically excluded during the early stages of the GC reaction before acquiring a centroblast phenotype. Furthermore, we provide evidence to indicate that an anergic response to $\mathrm{B}$ cell receptor stimulation may be responsible for such behavior. In contrast, in SLE, 9G4 B cells progressed unimpeded through this checkpoint, successfully participated in GC reactions, and expanded within the post-GC IgG memory and plasma cell compartments. The faulty regulation of $9 \mathrm{G} 4 \mathrm{~B}$ cells was not shared by RA patients. To our knowledge, this work represents the first comparative analysis of the fate of a specific autoreactive human $B$ cell population. The results identify a defective tolerance checkpoint that appears to be specific for human SLE.
\end{abstract}

\section{Introduction}

Systemic lupus erythematosus (SLE) is a prototypical autoimmune disease characterized by the production of antinuclear autoantibodies (1). SLE patients also frequently produce antilymphocyte antibodies of pathogenic potential that preferentially target CD45 $(2,3)$. These autoantibodies reflects a critical breakdown of B cell tolerance and the ensuing expansion of autoreactive $B$ cells capable of inducing disease through both antibody-dependent and antibody-independent mechanisms (4). Regrettably, understanding how tolerance is abrogated in SLE has been hampered by experimental limitations in identifying relevant autoreactive B cells.

We have proposed that B cells bearing the 9G4 idiotype (9G4 B cells) represent a useful system to study B cell tolerance in SLE, since the expression of this idiotype (which identifies antibodies encoded by the VH4.34 heavy chain) is synonymous with autoreactivity against $\mathrm{N}$-acetyllactosamine (NAL) determinants expressed by the iI blood group antigen and other self glycoproteins including CD45 (3, 5, 6). Moreover, the 9G4-determined autoreactivity is censored in normal subjects but greatly and specifically expanded in patients with active SLE. Thus, while 9G4 B cells represent 5-10\% of normal naive B cells, serum 9G4 antibodies are scarce in normal sera $(3,7)$. In contrast, these autoantibodies are abundant in patients with active SLE, representing $10-45 \%$ of total serum IgG $(3,8,9)$. Importantly, 9G4 antibodies represent the vast majority of anti-B cell CD45 antibodies and a significant fraction of anti-native double-stranded DNA (anti-dsDNA) antibodies, carry a disease specificity of $95 \%$, and correlate with disease activity and organ involvement $(3,8-10)$. These observations support the con-

Nonstandard abbreviations used: APC, allophycocyanin; BAFF, B cell-activating factor of the TNF family; BCR, B cell receptor; dsDNA, native double-stranded DNA; FDC, follicular DC; MZ, marginal zone; NAL, $N$-acetyllactosamine; SA, streptavidin; SLE, systemic lupus erythematosus.

Conflict of interest: The authors have declared that no conflict of interest exists.

Citation for this article: J. Clin. Invest. 115:3205-3216 (2005).

doi:10.1172/JCI24179. tention that 9G4 antibodies are of clinical relevance in SLE and that physiological tolerogenic mechanisms acting upon 9G4 B cells are defective in this disease.

Considering our demonstration that 9G4 B cells fail to form mature GCs in healthy subjects, we hypothesized that physiological mechanisms preventing the participation of these B cells in productive GC reactions were faulty in SLE (5). Alternatively, as shown for rheumatoid factor-transgenic B cells in autoimmune-prone mice, production of isotype-switched, somatically mutated autoantibodies can also originate outside the GC (11). Discriminating between these 2 possibilities requires the analysis of secondary lymphoid tissue, which is seldom available from SLE patients. We have overcome this obstacle by using tonsil biopsy samples from patients with SLE and RA controls. The results indicate that in SLE, but not in RA, 9G4 B cells escape normal censoring and actively participate in productive $\mathrm{GC}$ reactions, leading to the generation of substantially increased levels of IgG memory and plasma cells. The specific peripheral tolerance checkpoint that is overcome occurs at an early point in the GC reaction during the transition from the pre-GC to the centroblast stage, thus implicating faulty GC exclusion of autoreactive $\mathrm{B}$ cells in the pathogenesis of SLE.

\section{Results}

9G4 B cells are expanded in the GCs of SLE patients. Our previous studies of healthy individuals indicated that the vast majority of mature 9G4 B cells have a naive phenotype, fail to form GCs, and do not differentiate into mature bone marrow plasma cells. On that basis, we hypothesized that healthy autoreactive 9G4 B cells are barred from participating in productive GC reactions (5). In the present study, flow cytometry was used to compare the fate of 9G4 B cells in SLE tonsils $(n=6)$ and healthy tonsils $(n=15)$. As shown in Figure 1, most healthy 9G4 B cells (80-90\%) consistently expressed a naive $(\mathrm{Bm} 1 / \mathrm{Bm} 2)$ phenotype, failed to acquire a GC phenotype, and did not contribute significantly to post-GC IgG memory 

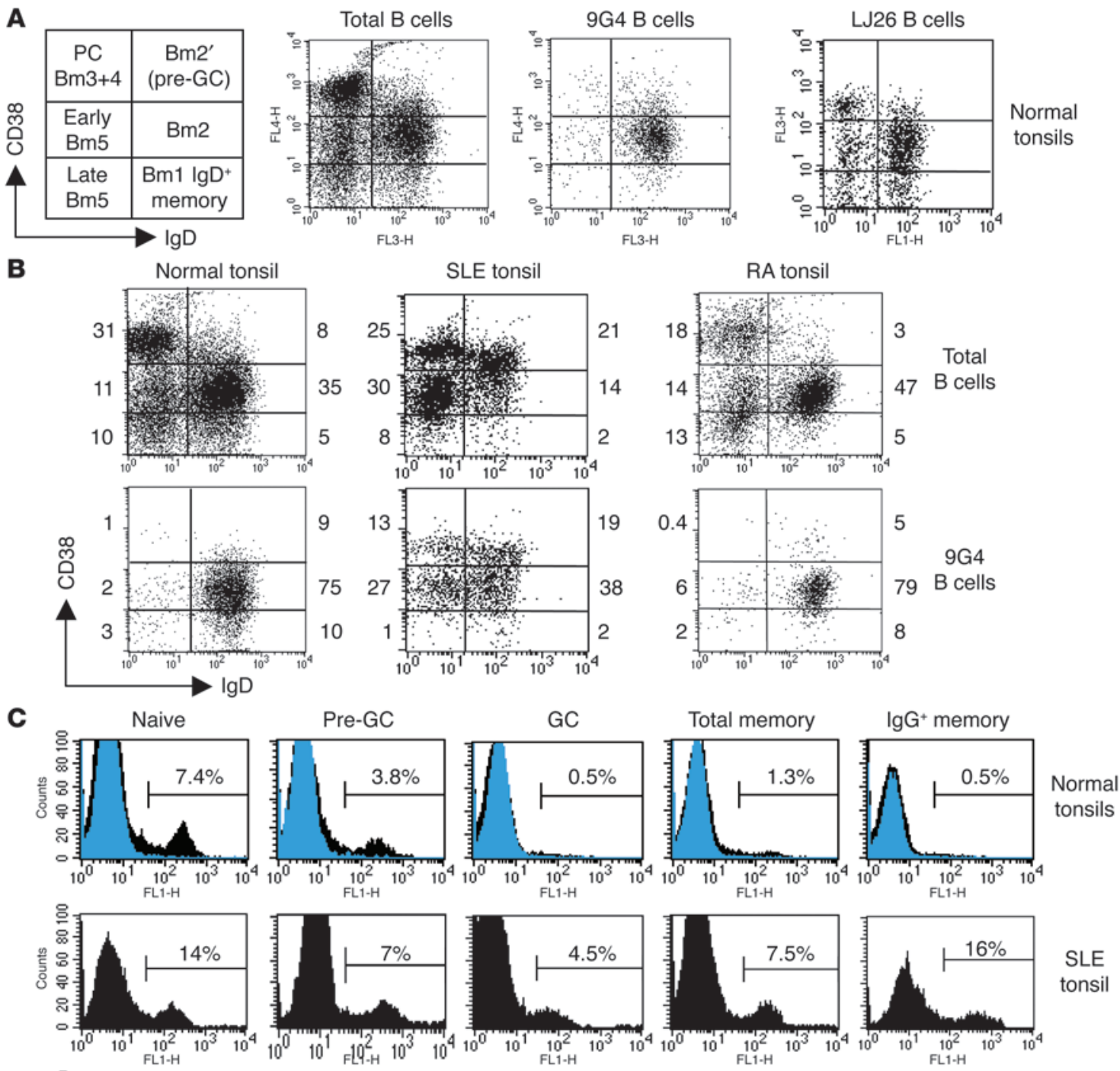

SLE

tonsil
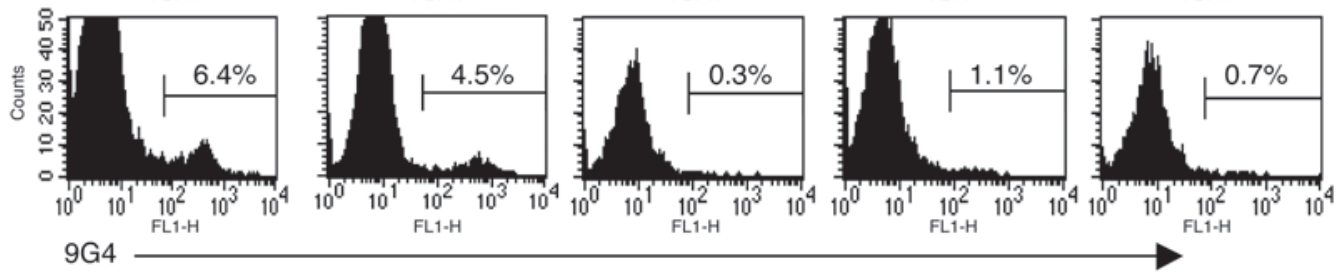

RA

tonsil

\section{Figure 1}

9G4 cells actively participate in GC reactions in SLE. (A) IgD/CD38 expression identifies the populations shown in the diagram on the left: IgD+CD38(Bm1 and lgD ${ }^{+}$memory); IgD+CD38+ $(\mathrm{Bm} 2)$; IgD ${ }^{+} \mathrm{CD}^{+} 8^{++}\left(\mathrm{Bm}^{\prime}\right.$ pre-GC cells); IgD-CD38++ (Bm3 and Bm4 or centroblasts and centrocytes); IgD-CD38 ${ }^{+}$ (early Bm5 memory); IgD-CD38- (late Bm5 memory); and CD38bright plasma cells (PC). Dot plots show the typical distribution of total, 9G4, and control VH3 (LJ26) B cells in normal tonsils. In every tonsil, 9G4 B cells were significantly underrepresented in the GC and memory compartments. (B) Tonsillar B cells from normal controls and SLE and RA patients analyzed as described above. Total tonsillar B cells demonstrate naive lymphopenia and expansion of pre-GC cells described in studies of SLE peripheral blood (68). In SLE, 9G4 B cells are not blocked at the pre-GC stage. Instead, they are greatly expanded in the GC and memory compartments. Yet these abnormalities are not found in tonsillar B cells from RA patients where 9 G4 cells are normally distributed. The percentage of each subpopulation is shown on the margins of the dot plots. (C) Histograms depict the frequency of $9 \mathrm{G} 4$ cells in normal, SLE, and RA tonsils. SLE patients show a large increase in the frequency of $9 \mathrm{G} 4 \mathrm{GC}$ cells and a 10- to 25 -fold increase in IgG 9G4 memory cells compared with healthy controls and RA patients. Blue histograms represent the staining obtained with the corresponding isotype control antibodies (data shown for normal controls), while 9 G4 histograms are depicted in black.

cells. In contrast, in SLE, 9G4 B cells were substantially represented among all mature B cell subsets (Figure 1B and Table 1). More specifically, SLE 9G4 B cells were significantly expanded within the GC and IgG memory compartments $(4.1 \% \pm 1.1 \%$ and $7.8 \% \pm 5.1 \%$ compared with normal frequencies of $0.4 \% \pm 0.15 \%$ and $0.6 \% \pm 0.3 \%$, respectively). 
Table 1

Frequency of $9 \mathrm{G} 4 \mathrm{~B}$ cells in tonsillar B cell subsets (\%)

\begin{tabular}{llcc}
\hline & & & \\
& Healthy & SLE & RA \\
Naive $(B m 1 / 2)$ & $7.2 \pm 2.1$ & $8.8 \pm 3.5$ & $6.7 \pm 1.3$ \\
Pre-GC (Bm2') & $4.8 \pm 2.0$ & $5.3 \pm 1.8$ & $4.2 \pm 1.8$ \\
GC (Bm3/4) & $0.4 \pm 0.2^{\mathrm{A}, \mathrm{B}, \mathrm{C}}$ & $4.1 \pm 1.1$ & $0.6 \pm 0.2^{\mathrm{D}, \mathrm{E}, \mathrm{F}}$ \\
Total memory & $1.8 \pm 0.7^{\mathrm{A}, \mathrm{B}, \mathrm{G}}$ & $5.5 \pm 2.3$ & $1.2 \pm 0.1^{\mathrm{D}, \mathrm{E}, \mathrm{H}}$ \\
IgG memory & $0.6 \pm 0.3^{\mathrm{A}, \mathrm{B}, \mathrm{C}, \mathrm{I}}$ & $7.8 \pm 5.1$ & $0.3 \pm 0.2^{\mathrm{D}, \mathrm{E}, \mathrm{F}, \mathrm{I}}$ \\
\hline
\end{tabular}

Relative representation of 9G4 B cells in the different B cell compartments in tonsils of healthy subjects and SLE and RA patients. The statistical significance of the differences found is indicated as follows: AP $<0.0001$ for GC, total memory, or IgG memory compared with naive in healthy subjects; $B P<0.0005$ for $\mathrm{GC}$, total memory, or IgG memory compared with pre-GC in healthy subjects; $C P<0.0001$ for $\mathrm{GC}$ or IgG memory, healthy compared with SLE; $D P<0.001$ for GC, total memory, or IgG memory compared with naive in $\mathrm{RA}$; $\mathrm{E} P<0.005$ for $\mathrm{GC}$, total memory, or IgG memory compared with pre-GC in $\mathrm{RA}$; ${ }^{\mathrm{F}} P<0.001$ for $\mathrm{GC}$ or IgG memory, RA compared with SLE; GP $<0.001$ for total memory, healthy compared with SLE; ${ }^{H} P<0.005$ for total memory, RA compared with SLE; IP $<0.005$ when IgG memory are compared to total memory in either healthy or RA. No significant differences were detected between the corresponding subsets in healthy subjects and RA patients.

Consistent with these results, parallel PBL studies showed that 9G4 IgG memory cells were increased 10- to 25 -fold over normal values (Figure 2A). Similarly, in contrast to their previously reported dearth among normal plasma cells (5), 9G4+ cells made up a substantial fraction of lupus plasmablasts (mean, $17 \%$ of 800 plasma cells analyzed; range, 13-19\%) (Figure 2B). In keeping with the 9G4 expansion observed within the IgG memory compartment, an average of $90 \%$ of all $9 \mathrm{G}^{+}$plasma cells were of the IgG isotype (as opposed to only $65 \%$ of total plasma cells; $P<0.001$ ), and $13 \%$ of all IgG plasma cells were $9 \mathrm{G}^{+}$(range, $10-22 \%$ ).

$G C$ exclusion of $9 G 4 B$ cells is preserved in $R A$. The abnormal developmental control of 9G4 B cells observed in SLE was not shared by patients with RA $(n=3)$, another systemic autoimmune disease in which B cells play an important pathogenic role but that is characterized by a different subset of autoantibodies and lack of serum 9G4 antibodies $(8,10)$. Instead, in RA patients, the distribution of tonsillar 9G4 B cells closely replicated a normal pattern, with preferential representation in the naive $\mathrm{Bm} 2 \mathrm{com}$ partment and a dramatic decline in the GC and post-GC compartments (Figure 1, B and C).

In healthy subjects, $9 \mathrm{G} 4 \mathrm{~B}$ cells are preferentially depleted in the postGC IgG memory compartment. The human memory B cell compartment is formed by both isotype-switched and nonswitched CD $27^{+}$ cells, with the latter subset formed largely by $\operatorname{IgD}^{+} \operatorname{IgM}{ }^{+}$cells (12). $\operatorname{IgD}^{+} \operatorname{IgM}^{+}$memory cells but not IgG memory cells develop normally in the absence of GCs, a fact that strongly suggests a GCindependent developmental pathway for the former subset (13). Consistent with this model and in keeping with their absence from the GC compartment, while the frequency of healthy $9 \mathrm{G} 4$ $\mathrm{B}$ cells among tonsillar total CD $27^{+}$memory B cells $(1.8 \% \pm 0.7 \%)$ was substantially lower than that among naive B cells, their abundance was particularly low among IgG memory cells, a population of GC origin (Figure 1C and Table 1).

Somatic hypermutation has the potential to abrogate the expression of the 9G4 idiotype in the GC and may indeed be an editing mechanism whereby a fraction of 9G4 GC cells might shed the 9G4-associated autoreactivity. Nevertheless, this mechanism is unlikely to be responsible for the observed loss of the 9G4 idiotype, since in healthy subjects, the majority of mutated VH4.34 B cells retain the $9 \mathrm{G} 4$ idiotype $(14,15)$. Moreover, if censoring were a result of simple loss of detection of a mutated 9G4 idiotype, then such censoring would be expected to occur in SLE, a disease where the activity of the mutational machinery may be enhanced (16). Furthermore, as shown in Figure 1A, a control idiotype (LJ26), which recognizes VH3-encoded antibodies and would be expected to be similarly disrupted by somatic hypermutation, is not lost during the GC reaction.

9G4 B cells accumulate within the spleen marginal zone. The analysis of healthy spleens $(n=7$; Figure $3 \mathrm{~A})$ confirmed the scarcity of $9 \mathrm{G} 4 \mathrm{~B}$ cells within the GC $(0.4 \% \pm 0.3 \%)$ and post-GC isotypeswitched memory populations $(0.5 \% \pm 0.2 \%$ and $0.74 \% \pm 0.35 \%$ of IgG and IgA memory cells, respectively) as compared with the naive and pre-GC compartments $(6.5 \% \pm 1.2 \%$ and $4.2 \% \pm 1.3 \%$,
Figure 2

9G4 B cells are expanded in the post-GC compartments. (A) FACS analysis of PBL CD19+ $B$ cells from active SLE patients stained with CD27, IgG, and 9G4 antibodies. A large increase in IgG $9 \mathrm{G} 4$ memory cells was demonstrated in SLE as compared with the very low levels typical of healthy subjects. Two representative SLE examples are shown. (B) For these same patients, CD138+ $\mathrm{PC}$ s were analyzed for cytoplasmic expression of the $9 \mathrm{G} 4$ idiotype and light chains or IgG. A large fraction of both total and IgG plasma cells in SLE were $9 \mathrm{G} 4^{+}$.
A

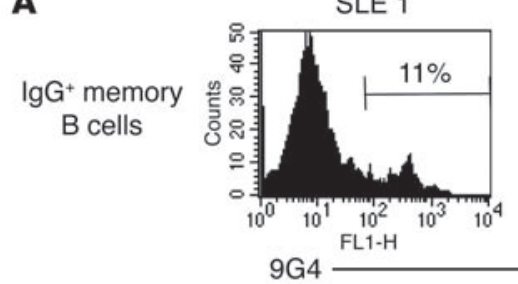

B

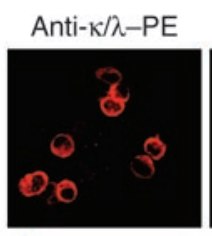

9G4-Alexa
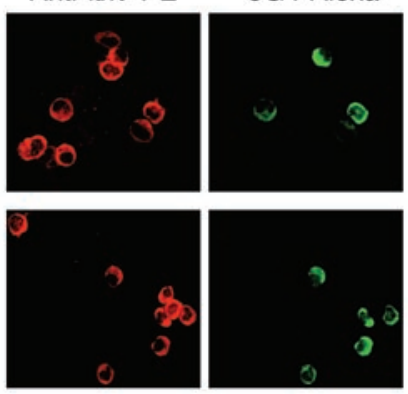
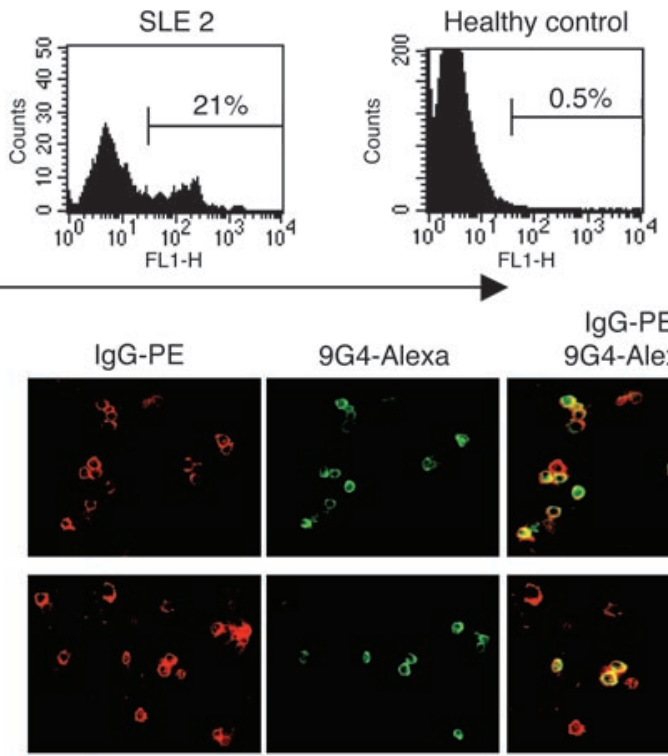

IgG-PE
9G4-Alexa

PCs

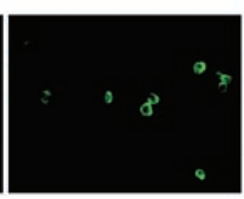

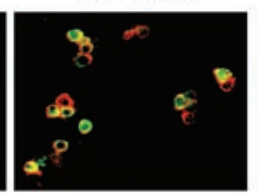

SLE 1

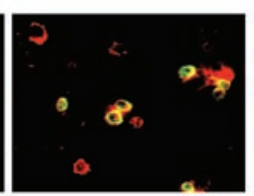


A

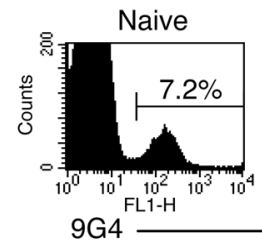

B
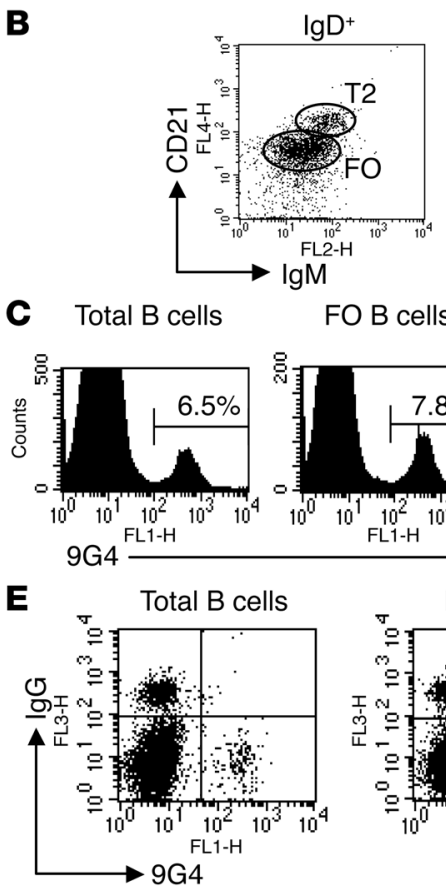

FO B cells
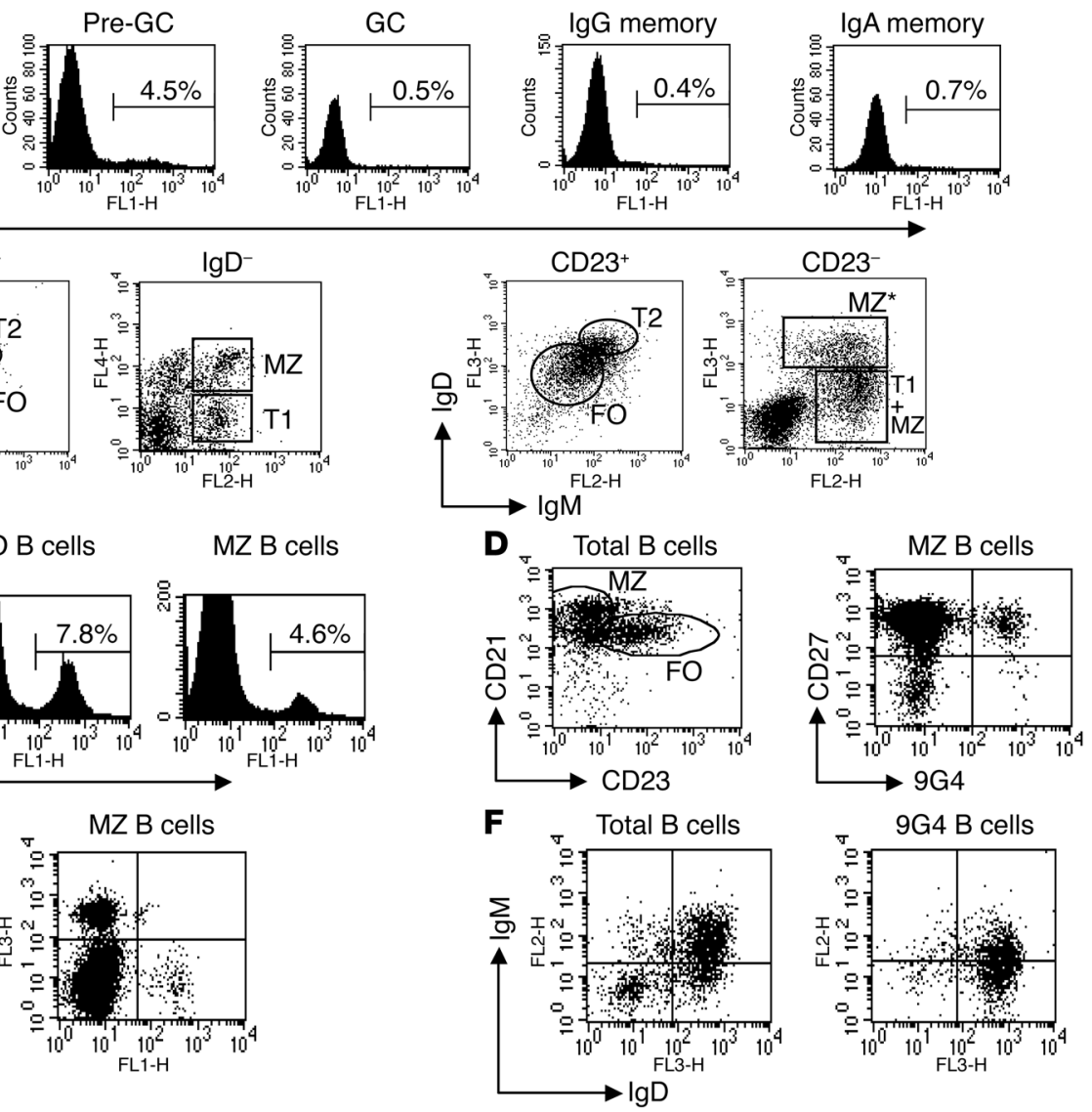

G

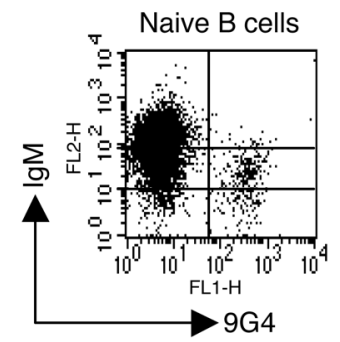

Total naive

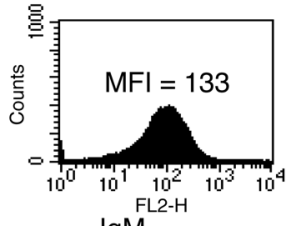

$\lg \mathrm{M} \stackrel{\mathrm{FL}-\mathrm{H}}{-}$ 9G4+ naive

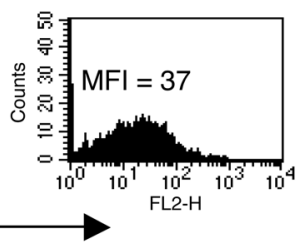

Figure 3

Analysis of 9G4 cells in healthy spleens. (A) CD19+ spleen B cells were analyzed with IgD, CD38, CD27, and 9G4 antibodies as described above ( $n=7$ spleens). 9G4 B cells are very scarce within the GC and post-GC compartments (IgG and IgA memory). Representative results are shown as histograms. (B) Staining for IgM, IgD, CD21, and CD23 expression identified transitional (T1 and T2), follicular (FO), and MZ populations with a distribution similar to mouse $B$ cells $(12,69)$. We identified an additional fraction composed of significant numbers of IgD+ cells, which represents a distinct subset of IgD+ $M Z B$ cells $\left(M Z^{*}\right)(19)$. (C) Total spleen B cells were fractionated into $M Z$ and follicular subsets as described above and further analyzed for the frequency of $9 G 4$ B cells. The frequency of spleen follicular $9 G 4$ cells was similar to the tonsil, and a lower but significant frequency was observed in the MZ fraction. (D) The majority of total and 9G4 MZ B cells express CD27. (E) The dearth of IgG and IgA 9G4 B cells was consistently documented in the spleen whether using total B cells or fractionated MZ B cells. (F) Dot plot analysis of total and $9 G 4$ spleen $B$ cells demonstrated that the vast majority of $9 G 4$ B cells express an IgM+lg $\mathrm{D}^{+}$phenotype. (G) Within the naive compartment, $9 \mathrm{G} 4 \mathrm{~B}$ cells express significantly lower levels of surface IgM. MFI, mean fluorescence intensity.

respectively). Interestingly however, $9 \mathrm{G} 4$ cells were substantially represented among marginal zone $(\mathrm{MZ}) \mathrm{B}$ cells $(4.8 \% \pm 1.4 \%)$, a population formed to a large extent by memory B cells including a subset bearing surface $\operatorname{IgM}$ and $\operatorname{IgD}$ with the ability to recirculate in the peripheral blood (Figure 3, B-F) (17-19). Furthermore, MZ 9G4 B cells predominantly expressed CD27, a marker of human memory B cells (Figure 3D). Yet, even within the MZ, 9G4 B cells were scarce among isotype-switched B cells (Figure 3, A, E, and F). Instead, 9G4 B cells almost universally expressed an $\operatorname{IgM}^{+} \mathrm{IgD}^{+}$sur- face phenotype whether in the tonsil, peripheral blood, or spleen (data shown for the spleen in Figure 3F).

Of note, this difference in 9G4 expression between IgM and IgG memory cells is consistent with the relative abundance of VH4-34 expression detected by PCR analysis of normal tonsillar $B$ cell subsets (14). Together, both studies indicate that while naive 9G4 B cells may differentiate into MZ IgM memory cells, presumably in a $\mathrm{T}$ cell-independent fashion, they do not participate in productive $\mathrm{GC}$ reactions required for the generation 
A

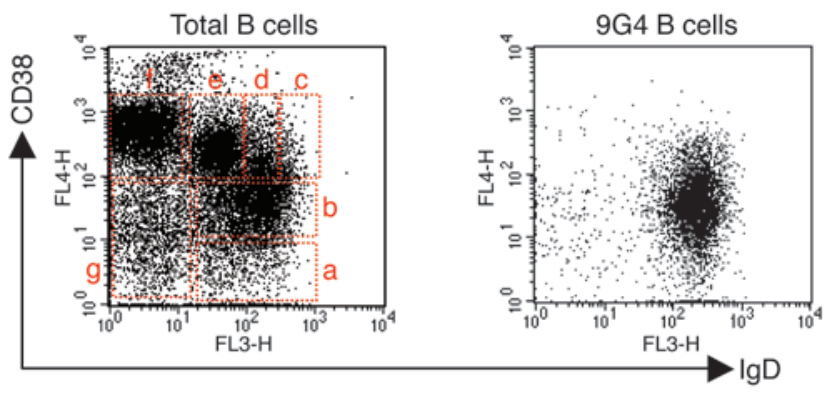

B
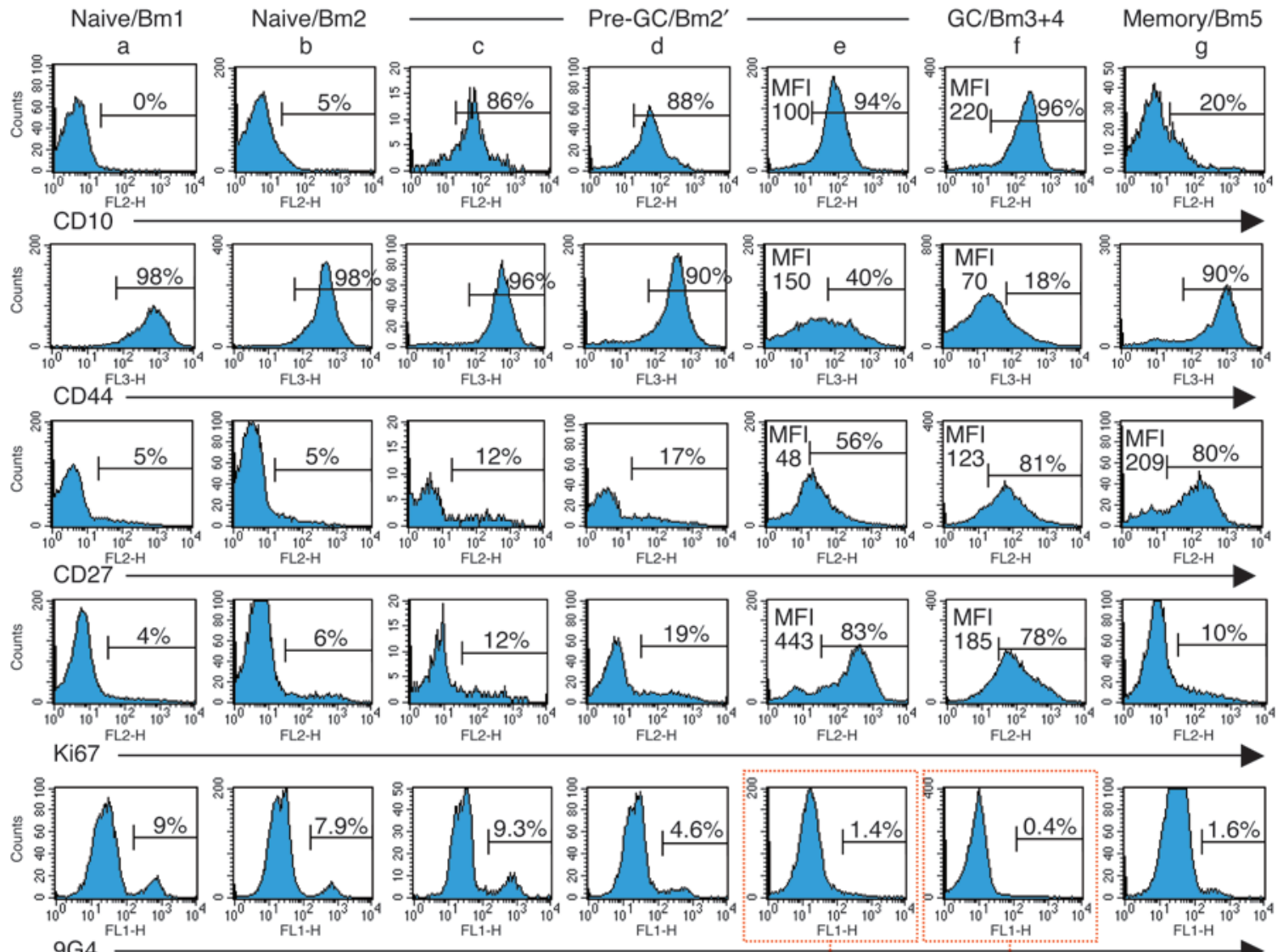

C
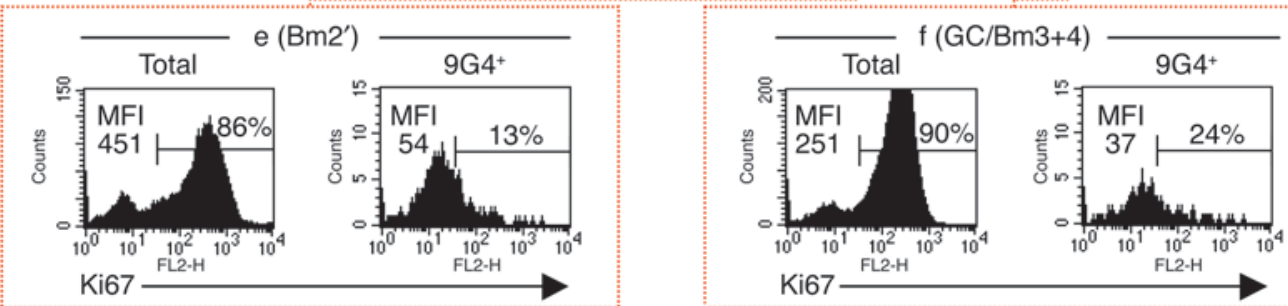

Figure 4

9G4 cells are normally censored at the GC founder stage. (A) The left dot plot is representative of normal tonsils, demonstrating a prominent $\mathrm{GC}$ founder population (fraction e). As shown in the dot plot on the right, even in these tonsils, $9 \mathrm{G} 4 \mathrm{~B}$ cells fail to progress past the pre-GC compartment and are scarce among GC founders. (B) Tonsils were analyzed for the expression of developmental markers CD10, CD44, and CD27 on conventional Bm1-Bm5 subsets (fractions a, b, f, and g). Pre-GC/Bm2' cells were further divided into 3 fractions (c-e), with e containing the putative GC founders. As shown in the corresponding histograms, CD10 (a GC marker) was progressively acquired in fractions c-f, while CD44 (a marker downregulated in GC) was progressively lost. CD27 also experienced progressive upregulation in fractions c-f. Strikingly, the highest expression of the nuclear proliferation protein Ki67 was observed in fraction e. These results are consistent with fraction e representing GC founders undergoing the initial phases of clonal expansion. Importantly, the majority of $9 \mathrm{G} 4 \mathrm{~B}$ cells was lost during pre-GC progression, greatly underrepresented among GC founders, and failed to expand within the GC, where their frequency continued to decline. (C) The scarce 9G4 B cells present within the GC founder and GC compartments were further analyzed for intracellular Ki67 expression. Consistent with their inability to form productive GC reactions, and in contrast to total B cells within these fractions, $9 \mathrm{G} 4$ B cells expressed very low levels of Ki67. 
A

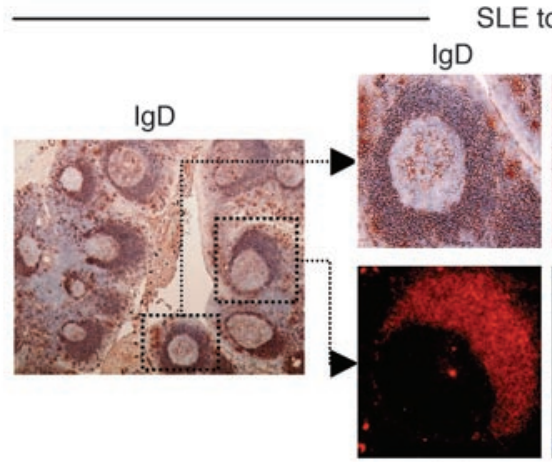

C

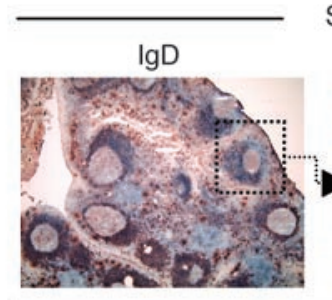

E
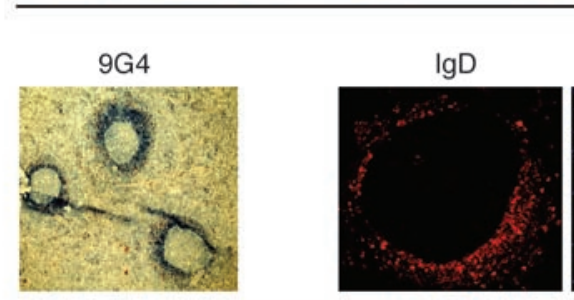

$\lg \mathrm{D}$

CD23/FDC

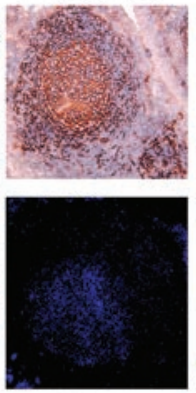

CD38

SLE tonsil

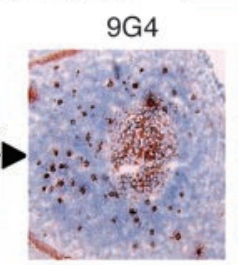

$\lg D$

LC1

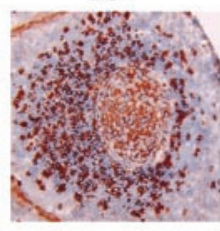

CD38
Ki67
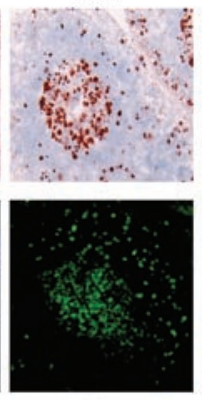

9G4
B

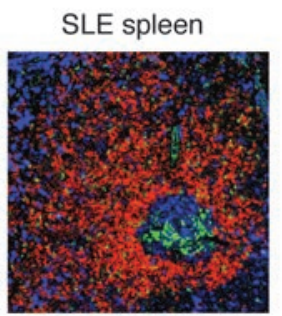

$\operatorname{lgD/CD38/9G4}$
9G4

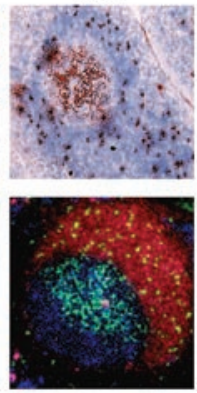

IgD/CD38/9G4

D

Healthy spleen

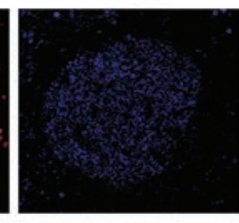

RA tonsil

CD20

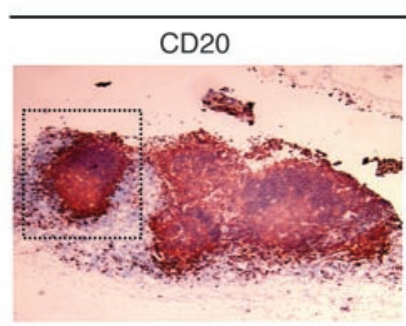

$\lg \mathrm{D}$

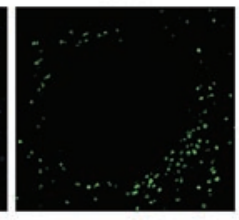

LC1/Ki67/lgD

9G4/Ki67/lgD
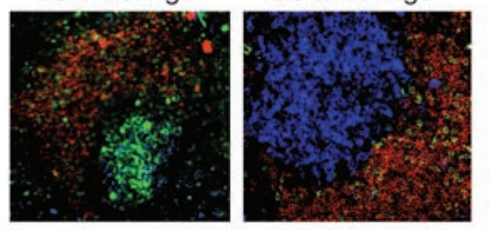

$\operatorname{lgD} / 9 \mathrm{G} 4$

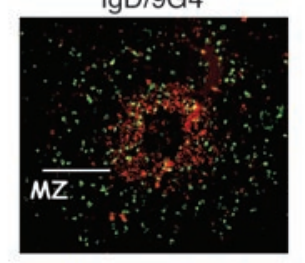

F

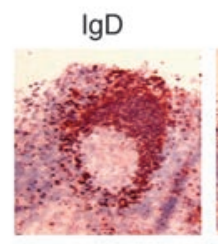

LC1

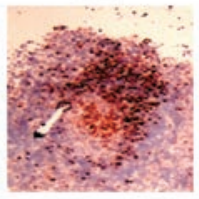

9G4

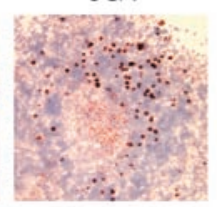

\section{Figure 5}

9G4 GCs are present in SLE patients at high frequency. (A) SLE tonsil biopsies stained with anti-lgD (left). Mature GCs formed by expansions of $9 G 4$ cells were frequently identified in SLE. The upper row of the enlarged images depicts a typical mature $9 \mathrm{G} 4^{+}$proliferative GC with a wellformed FDC network (CD23/FDC) and positive Ki67 staining. The lower row shows similar findings by immunofluorescence: follicular mantle (IgD-PE, red), GC (CD38-7-aminomethylcoumarin, blue), and 9G4 (Alexa 488, green). (B) Representative example of a 9G4+ GC in an SLE spleen shown by 3-color immunofluorescence. (C) SLE GC showing the expansion of both 9G4 and LC1 B cells. (D) Healthy tonsils stained by immunofluorescence: IgD (red), Ki67 (blue), and either 9G4 or LC1 (both green). In contrast to SLE, healthy tonsils lack 9G4+ GC. Yet proliferating LC1 GCs were readily demonstrated. (E) A representative field from healthy spleens demonstrating the absence of 9 G4 staining in the GCs is shown on the left. These results were routinely corroborated by immunofluorescence (middle panels). The photograph on the right illustrates the absence of $9 \mathrm{G} 4 \mathrm{~B}$ cells from the GC and their accumulation within the FO and the MZ. (F) Enzymatic staining of serial sections obtained from tonsil biopsies of patients with RA failed to demonstrate 9G4+ GCs. Instead, as in healthy subjects, 9G4 B cells were restricted to the follicular mantle. In contrast, $\mathrm{LC1}^{+} \mathrm{GCs}$ were readily identified in these patients.

of IgG memory cells. Indeed, our results lend further support for the existence of a GC-independent developmental pathway leading to the generation of $\operatorname{IgM}^{+} \operatorname{IgD}^{+}$memory cells but not IgG memory cells $(13,19)$.

Development of healthy $9 \mathrm{G} 4 \mathrm{~B}$ cells is blocked at the GC founder stage. In order to specifically pinpoint the checkpoint(s) responsible for the GC exclusion of 9G4 B cells in healthy subjects, we conducted detailed tracking studies of these cells throughout the pre-GC compartment. As illustrated in Figure 4, the pre-GC/Bm2' popu- lation can be further divided into several fractions that gradually lose surface IgD expression while acquiring GC markers including CD10. Analysis of these fractions allowed us to define a subset that expresses low levels of surface IgD (intermediate between pre-GC and centroblast levels) and the highest levels of Ki67 among all tonsillar B cells, including centroblasts.

Our results suggest that cells of this fraction, which can be visualized as a distinct population in about $25 \%$ of normal tonsils (shown in Figure 4A), are the earliest identifiable GC cells and are 


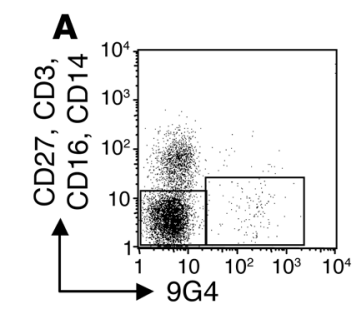

\section{B}

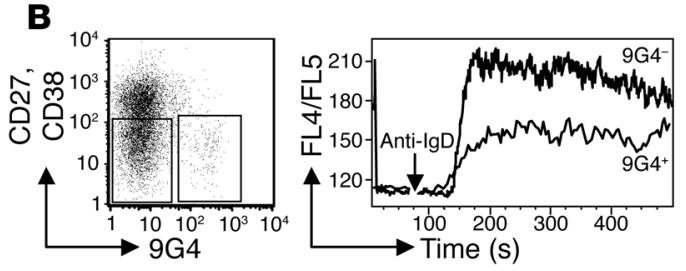

D

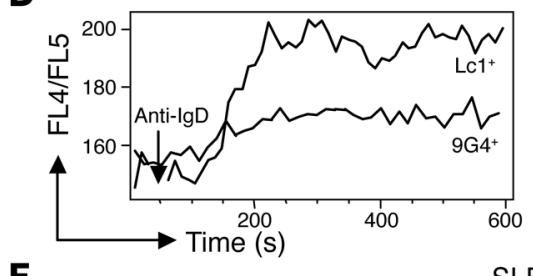

$\mathbf{F}$

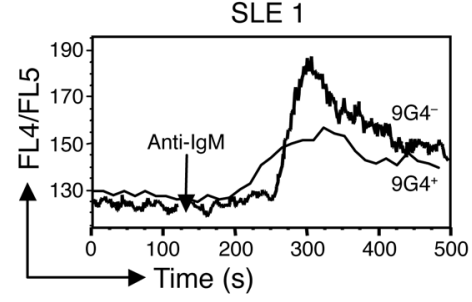

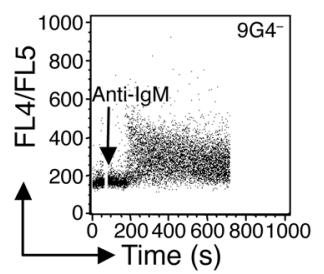
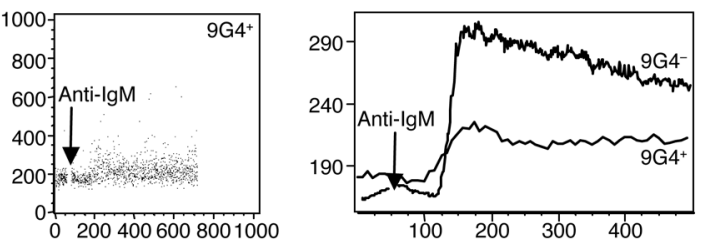

\section{C}
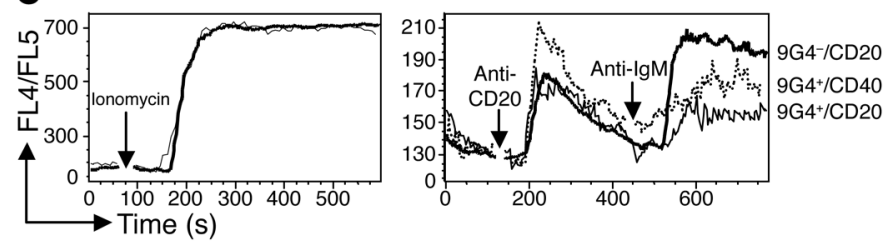

$\mathbf{E}$

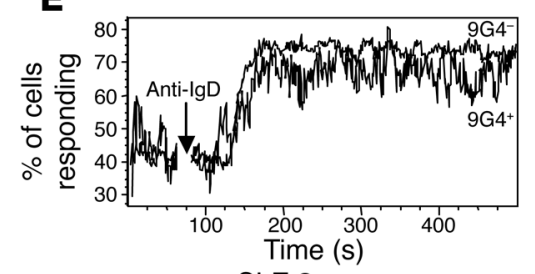

SLE 2

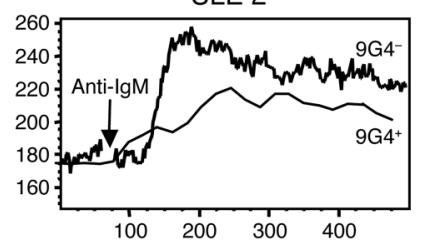

\section{Figure 6}

9G4 B cells display attenuated calcium responses. (A) Calcium responses in healthy PBL B cells after anti-IgM stimulation. B cells were purified by negative selection and stained with CD27-, CD3-, CD14-, and CD16-PE antibodies (to gate out non-B cells and memory B cells) and 9G4 at the time of calcium measurements (gating shown on the left). The responses of individual cells are depicted in the middle dot plots, and the median responses in the graphs at right. (B) Calcium responses in tonsillar naive B cells after anti-lgD stimulation. Cells were stained with CD38 mAb and CD27 mAb to gate out memory and GC B cells, as well as $9 \mathrm{G} 4$ at the time of calcium measurements. The attenuated response of $9 \mathrm{G} 4{ }^{+}$cells is not due to IgM downmodulation, since similar results were seen with anti-lgD. (C) 9 G4 B cells normally mobilize calcium in response to ionomycin and CD20 cross-linking. Yet anti-CD20 failed to restore full signaling upon subsequent BCR stimulation. Of note, CD40 costimulation by overnight incubation with CD40 ligand-expressing fibroblasts (dotted line) increased the response through both CD20 and the BCR, yet it failed to fully restore BCR signaling to the same level seen for 9G4- cells. (D) 9 G4 naive B cells have attenuated signaling compared with another positively selected naive B cell population. 9G4 tonsillar B cells were purified by magnetic selection using 9G4-FITC. Control VH4 naive B cells were purified using LC1. Calcium responses in the naive gated population were examined as described above. (E) The percentage of cells responding to BCR stimulation was similar in $9 \mathrm{G} 4^{+}$cells as compared with naive control B cells, indicating that the differences shown in $\mathbf{D}$ were due to lower average response per cell. (F) Calcium signaling through the BCR is similarly attenuated in PBL naive 9G4 B cells from SLE patients.

likely to represent GC founders. Of note, approximately $90 \%$ of all 9G4 B cells fail to acquire this pre-centroblast, GC founder phenotype (Figure 4B). Strikingly, while the majority of all GC founders and GC cells express high levels of Ki67, the residual 9G4 B cells found within these compartments expressed very low levels of this proliferation nuclear protein (Figure 4C). Together, these data indicate that the main censoring of autoreactive 9G4 B cells occurs during the early phases of the GC reaction, with relatively few $9 \mathrm{G} 4$ $\mathrm{B}$ cells differentiating into GC founders. Moreover, residual 9G4 B cells with a GC founder or a centroblast phenotype appear unable to proliferate and do not develop into more mature centrocytes.

Histological studies of $9 G 4 \mathrm{~B}$ cells in SLE patients and normal subjects. The participation of 9G4 B cells in mature GC reactions in SLE was confirmed histologically. Proliferating Ki67+ 9 G4 B cells con- tributed to $18 \%$ of 160 mature GC from 6 SLE tonsils (Figure 5, A and C). Similar findings were also obtained with 2 SLE spleens (Figure 5B). The interpretation of these results may be confounded by retention of circulating 9G4 antibodies by follicular DCs (FDCs). However, we believe that the actual presence of 9G4 B cells in the GC, as indicated by the FACS results, is strongly supported by costaining with B cell GC markers such as CD38. Furthermore, FDC-bound 9G4 antibody would be expected to produce staining of most GC and comparable staining of neighboring GC of similar age as indicated by well-developed FDC networks. Such findings, however, were not observed.

This abundance of $9 \mathrm{G} 4^{+} \mathrm{GC}$ in SLE is in stark contrast to the scarcity of similar structures in healthy subjects. Thus far, we have analyzed 705 secondary follicles (tonsils: 500 follicles, $n=15$; 
adult spleens: 120 follicles, $n=7$; infant spleen: 85 follicles, $n=1$ ) and found only 1 instance of a GC formed by expansion of 9G4 B cells in the infant spleen (data not shown). Instead, as shown by triple immunofluorescence, in healthy subjects, 9G4 B cells were predominantly localized both in tonsils and spleens within the follicular mantle (Figure 5, D and E). In addition, scrutiny of the well-developed spleen MZ confirmed the FACS data by showing that $9 \mathrm{G} 4 \mathrm{~B}$ cells are substantially represented within this anatomical compartment (Figure 5E).

In contrast to $9 \mathrm{G} 4 \mathrm{~B}$ cells, control B cells expressing antibodies encoded by other VH4 gene family members (as recognized by the antiidiotypic mAb LC1) did form mature proliferating GCs with the same frequency in normal subjects and SLE patients (38\% and $40 \%$, respectively) (Figure 5, C and D, and data not shown). The high frequency of $\mathrm{LC}^{+} \mathrm{GCs}$ would be expected in the absence of significant counterselection because $\mathrm{LC}^{+} \mathrm{VH} 4$ cells represent approximately $20 \%$ of all naive B cells, and GCs usually contain the progeny of $3-5$ founder cells $(20,21)$. The abundance of $\mathrm{LC}^{+}{ }^{+} \mathrm{GCs}$ provides a meaningful counterpoint to the scarcity of $9 \mathrm{G} 4^{+} \mathrm{GCs}$ and represents a useful estimate of the magnitude of negative selection experienced by 9G4 B cells. Considering that VH4.34 B cells represent $30-50 \%$ of all naive $\mathrm{VH} 4$ cells $(20,22)$, in the absence of negative selection, $12-25 \%$ of all GCs would be expected to be $9 \mathrm{G}^{+}$, a figure that is consistent with our findings in SLE patients. Instead, our analysis of large numbers of GCs from multiple donors indicates that the frequency of such events must be well below $1 \%$. This striking scarcity of mature $9 \mathrm{G}^{+} \mathrm{GCs}$ is in keeping with the experience of other investigators (J. Spencer and D. Dunn-Walters, Guy's, King's and St Thomas' Medical School, London, United Kingdom; personal communication), who have failed to observe any such structures in the course of extensive studies of human GCs (17). Consistent with the oligoclonal composition of mature GCs, approximately $30 \%$ of all SLE LC1 ${ }^{+}$GCs also contained expansions of 9G4 cells, an event never identified in healthy subjects (Figure 5C).

Finally, histological analysis of tonsil specimens obtained from RA patients $(n=3)$ demonstrated that, much like in healthy controls, 9G4 cells were anatomically localized to the follicular mantle and absent from the GC proper (Figure 5F).

$9 \mathrm{G} 4 \mathrm{~B}$ cells display an anergic phenotype. As a first step to explain the failure of naive $9 \mathrm{G} 4 \mathrm{~B}$ cells to proliferate and form productive GCs in healthy subjects, their response to B cell receptor (BCR) stimulation was analyzed using intracellular calcium flux as a global readout of proximal signaling. Both tonsillar and peripheral blood B cells were used for these experiments and consistently showed markedly decreased $\mathrm{Ca}^{2+}$ flux as compared with control naive B cells (Figure 6, A-E). Despite their diminished response to BCR stimulation, 9G4 B cells responded normally to stimulation with either calcium ionophores or through CD20, a putative calcium channel. Yet initial stimulation through either CD20 or CD40 failed to restore full response upon subsequent $\mathrm{BCR}$ stimulation (Figure $6 \mathrm{C}$ ). Interestingly, naive 9G4 B cells obtained from the same SLE patients in whom positive $9 \mathrm{G} 4 \mathrm{GC}$ had been identified through tonsil biopsy also displayed attenuated $\mathrm{Ca}^{2+}$ responses after BCR stimulation, as shown in Figure 6F.

These attenuated responses are reminiscent of anti-HEL and anti-dsDNA transgenic B cells which are rendered anergic by chronic stimulation by the corresponding self antigens $(23,24)$.
As in these models, an anergic phenotype is also supported by the expression of low levels of surface IgM in 9G4 as compared with control naive B cells (shown in Figure 3G).

\section{Discussion}

To the best of our knowledge, our results represent the first analysis of the comparative fate of a discreet population of mature autoreactive $\mathrm{B}$ cells of pathogenic potential in healthy and autoimmune human subjects. Our work also provides some of the first insights into the mechanisms of human peripheral tolerance. In its totality, it demonstrates that physiological mechanisms that prevent autoreactive naive follicular B cells of pathogenic potential from participating in a productive GC reaction are faulty in SLE. This failure is not shared by patients with RA, and therefore, at least for this particular subset of autoreactive B cells, the underlying tolerance defect appears to be specific for SLE.

The human mature B cell repertoire is highly populated by autoreactive $B$ cells of pathogenic potential as illustrated by 9G4 B cells (5). More recently, it has been shown that despite the presence of earlier checkpoints acting upon immature and transitional B cells, up to $20 \%$ of all human naive B cells produce autoantibodies that prominently include antinuclear antibodies (25). In addition, 2 articles published during the preparation of this manuscript suggest that patients with SLE and RA are defective in censoring transitional autoreactive B cell $(26,27)$. In spite of the fact that the mature autoimmune repertoire is quite different between SLE and RA, no significant differences were detected in the antigenic autoreactivity of the mature $\mathrm{B}$ cells analyzed in these patients. These results suggest that, as illustrated by the specificity of 9G4 B cells for SLE, subsequent events are critical in selecting the autoimmune repertoire characteristic of these diseases. Hence, the available evidence strongly indicates a need for effective censoring of mature B cells in order to maintain peripheral tolerance. The work presented here supplies evidence in that regard.

How are $9 \mathrm{G} 4 \mathrm{~B}$ cells censored in bealthy subjects? In order to prevent autoimmunity, censoring mechanisms, including anergy and sequestration into the $\mathrm{MZ}$, ultimately forbid the participation of mature autoreactive $B$ cells in productive GC reactions, thereby precluding their expansion into the long-lived IgG memory and plasma cell compartments. However, some autoreactive B cells may still filter through these pre-GC checkpoints and initiate $\mathrm{GC}$ reactions. Moreover, autoreactivity can arise de novo in the $\mathrm{GC}$ through somatic hypermutation $(28,29)$. Both these possibilities create the need for specific GC reaction checkpoints and, indeed, GC censoring has been demonstrated in different mouse models (29-34).

The failure of healthy $9 \mathrm{G} 4 \mathrm{~B}$ cells to generate productive GC reactions could be due either to inability to initiate a $\mathrm{GC}$ reaction (GC exclusion) or to active censoring during ongoing $\mathrm{GC}$ reactions (GC censoring). Our current results favor GC exclusion, since naive 9G4 B cells readily acquire a pre-GC phenotype, yet the majority of them fail to develop into GC founders (Figure 4). Yet a significant frequency of $9 \mathrm{G} 4 \mathrm{~B}$ cells is present in the GC founder population. Importantly, neither $9 \mathrm{G} 4 \mathrm{~B}$ cells with a founder phenotype nor the rare $9 \mathrm{G} 4$ cells that acquire a GC phenotype are able to proliferate and form mature GC reactions. It is therefore plausible that a fraction of 9G4 B cells could escape GC exclusion at the main pre-GC checkpoint and experience active GC censoring, as recently shown for murine anti-DNA B cells (33). 
Our results indicate that anergy plays a major role in the censoring of 9G4 B cells, and it may provide a unifying mechanism to explain the inability of these cells to generate either antibodyforming cells or GCs. In several transgenic models, anergy is associated with developmental arrest, follicular exclusion, and premature death $(24,35)$. In other transgenic systems, autoreactive B cells avoid anergy and develop into mature follicular B cells but are ultimately excluded from the GC pathway either by clonal ignorance or by learned ignorance, in the latter case due to progressive developmental downregulation of both surface $\operatorname{IgM}$ and $\operatorname{IgD}(36,37)$. The behavior of 9G4 B cells departs from these models in several respects since they display an anergic phenotype characterized by low IgM levels (but normal IgD) and greatly attenuated calcium flux in response to BCR stimulation, yet they develop into mature $\mathrm{B}$ cells both in the follicular and the long-lived MZ compartments (18). Interestingly, similar uncoupling of anergy and developmental arrest has been described for anti-insulin transgenic B cells, which can also accumulate within the MZ (38). Moreover, as it is the case for $9 \mathrm{G} 4$ cells in SLE, the attenuated BCR response of antiinsulin B cells is maintained in NOD mice (38). Yet a critical difference is that anti-insulin B cells do not participate in GC reactions, even in an autoimmune background where they contribute to the induction of autoimmune diabetes.

It is important to bear in mind that while anergy could be induced by exposure to self antigen within the follicle (such as CD45 and other NAL-bearing glycoproteins), it could also be imprinted in 9G4 B cells at earlier developmental stages, as shown for anti-p-azophenylarsonate B cells in transgenic mice (39). In these mice, low-intensity chronic stimulation received at the immature stage in the bone marrow is sufficient to induce full maturation in the spleen while maintaining a refractory response to antigenic stimulation (39). In addition to NAL-bearing glycoproteins expressed by different cell lineages, red blood cells would also provide an abundance of antigen capable of engaging immature 9G4 B cells in the bone marrow.

Importantly, an anergic response to antigen engagement could facilitate GC exclusion by impairing the ability of 9G4 B cells to appropriately respond to cognate $\mathrm{T}$ cell help, by rendering these cells unable to effectively compete for B cell-activating factor of the TNF family (BAFF) or by increasing sensitivity to Fas-mediated deletion (40-43). These mechanisms need not be mutually exclusive and may well work synergistically. Interestingly, both absence of $\mathrm{T}$ cell help and defective BAFF-mediated signaling may also result in unsuccessful GC reactions (44-47). Therefore, these mechanisms could also contribute to GC censoring of $9 \mathrm{G} 4$ B cells that escape GC exclusion.

The abundance of MZ 9G4 B cells suggests that their exclusion from the GC could also result from being selected into a GC-independent, T cell-independent developmental pathway. While it is formally possible that such commitment could take place after 9G4 B cells acquire a pre-GC phenotype, most of the available evidence indicates that $\mathrm{MZ} \mathrm{B}$ cells originate from transitional precursors (48). Therefore, our results can be reconciled with current models of $\mathrm{B}$ cell development by proposing the existence of separate subsets of transitional 9G4 B cells with the potential to differentiate into either follicular or MZ B cells on the basis of distinct antigenic reactivity and different $\mathrm{BCR}$ signaling strength (49). Under this model, transitional 9G4 B cells endowed with attenuated or abolished autoreactivity, possibly through receptor editing, would be selected into the MZ (50). A similar binary model of autoreactivity and selection could also be operative in follicular B cells, which may also have the potential to differentiate into MZ B cells (18). These models are currently being investigated in our laboratory by determining the antigenic reactivity of 9G4 B cells in the corresponding compartments.

How is tolerance subverted in SLE? It is apparent that the strength and quality of signaling through the BCR inform most of the decisions made by B cells $(41,49,51)$. Consequently, the tolerance breakdown of $9 \mathrm{G} 4 \mathrm{~B}$ cells in SLE could have been simply explained by breach of anergic responses to BCR stimulation. Theoretically, this could occur even at early developmental stages, and indeed, such a mechanism could be invoked to explain the observation that censoring of autoreactive transitional $\mathrm{B}$ cells may be defective in SLE (27). However, in contrast to the persistence of 9G4 B cells observed in mature, long-lived compartments, censoring of transitional B cells seems to be mediated by deletion. Furthermore, our data show that, like in healthy subjects, lupus naive 9G4 B cells capable of generating mature $\mathrm{GC}$ reactions maintain attenuated responses to BCR stimulation. This observation suggests that the breakdown of tolerance observed in SLE is due either to abnormal extrinsic costimulation or abnormal intrinsic response of $9 \mathrm{G} 4 \mathrm{~B}$ cells to T celldependent or -independent stimuli or to survival factors. Thus, it is possible that, as demonstrated in different animal models, abnormal $\mathrm{T}$ cell help and/or defective $\mathrm{T}$ regulatory activity might contribute to the successful participation of $9 \mathrm{G} 4 \mathrm{~B}$ cells in productive GC reactions in SLE patients $(52,53)$.

$\mathrm{T}$ cell-independent costimulation of $\mathrm{B}$ cells through TLRs has the potential to overcome B cell tolerance and could participate in the deregulation of 9G4 cells in SLE (54). Significantly, at least a fraction of $9 \mathrm{G} 4 \mathrm{~B}$ cells recognize antigens capable of engaging 1 or more members of the expanding family of TLRs. Such antigens include LPS (for TLR4 and RP105) and DNA (for TLR9) (55). Furthermore, our preliminary results indicate that 9G4 antibodies may bind to apoptotic cells, which could provide costimulation through either TLR4 or TLR9 (56-58). Apoptotic bodies, which may not be readily available in healthy subjects due to highly efficient clearance mechanisms, have been shown to accumulate in SLE GC due to deficient clearance and could supply the necessary antigenic drive for 9 G4 B cells $(59,60)$. Such reactivity could both explain the participation of lupus 9G4 B cells in GC reactions and define a pathogenic role for 9G4 antibodies, since lupus IgG complexed to apoptotic cells stimulate the production of IFN- $\alpha$, and 9G4 antibodies constitute a substantial fraction of lupus serum $\operatorname{IgG}(3,61)$.

As previously discussed, insufficient BAFF signaling may contribute to the censoring of anergic $\mathrm{B}$ cells, and interestingly, the behavior of 9G4 B cells is reminiscent of mouse GC B cells defective in BAFF-receptor signaling, which are characterized by an autonomous proliferative defect with reduced Ki67 expression and inability to sustain productive GC reactions $(45,46)$. Conversely, excessive BAFF stimulation may overcome B cell anergy and induce systemic autoimmunity $(43,62,63)$. Given that human SLE is characterized by increased BAFF levels, it is tempting to speculate that this abnormality might contribute to the breakdown of tolerance observed in lupus 9G4 B cells (64).

In summary, our work demonstrates the existence of a defective checkpoint in the maintenance of peripheral B cell tolerance that appears to be specific to patients with SLE. It also shows that the anergic phenotype of healthy $9 \mathrm{G} 4 \mathrm{~B}$ cells is not overcome 
simply by intrinsic BCR hyperresponse of SLE B cells but that additional extrinsic costimulation must be at play. Additional cellular, biochemical, and genetic analysis of 9G4 B cells, currently underway in our laboratory, and a better understanding of the antigen-binding properties of $9 \mathrm{G} 4$ antibodies should shed considerable light on the mechanisms responsible for the breach of tolerance observed in SLE.

\section{Methods}

Human samples. Samples were obtained with informed consent using protocols approved by the University of Rochester Medical Center Institutional Review Board. Peripheral blood and tonsils were obtained as previously described (5). SLE patients were selected if they had a diagnosis of SLE with at least 4 American College of Rheumatology (ACR) criteria, had a SLE disease activity index of at least 10 , and were only treated with antimalarials and/or low-dose prednisone ( $<10 \mathrm{mg} / \mathrm{d}$ ). RA patients were recruited if they had a clinical diagnosis of RA and fulfilled ACR criteria (65). Tonsil samples were acquired from consenting SLE and RA patients by triangular adenoid forceps biopsy. Spleens were obtained from healthy individuals with traumatic spleen rupture and from 2 SLE patients at autopsy. A spleen from a 2-year-old girl who died from an accidental fall was obtained from the National Disease Research Interchange.

$B$ cell isolation. PBMCs were isolated by gradient centrifugation at $4{ }^{\circ} \mathrm{C}$ using Ficoll-Paque (Amersham Biosciences). PBL B cells were obtained through magnetic positive selection using CD19 microbeads (MACS; Miltenyi Biotec) with a final purity of greater than $98 \%$. Splenic and tonsillar B cell suspensions were generated, and $\mathrm{T}$ cells depleted using 2-aminoethylisothiouronium bromide-sheep red blood cells (2-AETSRBC; Colorado Serum Co.) (5). The resulting cells (>97-99\% CD19+) were directly analyzed by flow cytometry.

FACS analysis. Single-cell suspensions $\left(10^{6} /\right.$ sample) were labeled at $4^{\circ} \mathrm{C}$ with predetermined optimal concentrations of fluorophore-conjugated $\mathrm{mAbs}$ and pair-matched isotype controls. The following antibodies were used: anti-CD19-allophycocyanin (anti-CD19-APC) (SJ25C1), antiCD27-PE (L128), streptavidin-peridinin chlorophyll protein, rat IgG2aFITC (isotype control for 9G4) (BD); biotinylated anti-IgD and anti-IgDFITC (IA6-2), anti-IgM-PE (G20-127), biotinylated anti-IgG (G18-145), anti-CD10-PE (HI10a), anti-CD21-PE or -APC (B-1Y4), anti-CD23-PE (M-L233) or -APC (EBV-CS-5), anti-CD38-APC (H1T2), biotinylated anti-CD44 (G44-26), anti-CD77-FITC (5B5), and anti-Ki67-PE (B56; BD Biosciences - Pharmingen). The 9G4 mAb was kindly provided by F.K. Stevenson (Tenovus Research Laboratories, Birmingham, United Kingdom) and recognizes a framework 1 region-encoded idiotype that is expressed by all unmutated and close to $90 \%$ of mutated VH4.34 B cells present in the normal repertoire $(14,15)$. Control VH3 B cells were detected with the antiidiotypic mAb LJ26 (66). All samples were analyzed via FACSCalibur using CellQuest software version 3.3 (BD Biosciences). In total, 50,000-100,000 events, gated for live B lymphocytes, were collected for each sample. Statistical significance was assessed using nonparametric Mann-Whitney $U$ test with GraphPad Prism software version 3.

Plasma cell analysis. $\mathrm{CD} 138^{+}$peripheral blood plasma cells were analyzed by intracellular staining as previously described (5). Briefly, $10^{5}$ cells were cytospun, fixed with $2 \%$ paraformaldehyde, and labeled with biotinylated 9G4/SA-Alexa 488 (SA, streptavidin) (Invitrogen Corp.) and either anti- $\mathrm{\kappa} / \lambda \mathrm{F}\left(\mathrm{ab}^{\prime}\right)_{2}-\mathrm{PE}$ (SouthernBiotech) or anti-IgG-PE (BD Biosciences - Pharmingen). Slides were analyzed using an Olympus BX40 microscope with a BX-FLA Mercury source reflected light fluorescence attachment. Acquired images were overlaid using Image-Pro software (version 4.1; MediaCybernetics). Plasma cell morphology was also assessed by Giemsa staining.
Immunohistochemistry studies. Serial tonsil sections were stained using a Dako LSAB2 System (DakoCytomation). Tonsil tissue was flash frozen in OCT, and 4- $\mu \mathrm{m}$-thick acetone-fixed cryostat sections were incubated at room temperature for 60 minutes with primary antibodies (antiCD20 antibody [Dako N1502], anti-CD3 antibody [Dako M0835], 9G4 or LC1, anti-IgD, anti-CD23 [Dako M4M6], Ki67 [Dako Ki-S5], antiFDC [Dako CNA-42]). The mouse mAb LC1 recognizes a subset of VH4 genes that does not include VH4.34, in a mutually exclusive fashion with $9 \mathrm{G} 4$ (67). After rinsing with $\times 1$ PBS, sections were incubated for 30 minutes at room temperature with biotinylated anti-mouse or antirat Ig secondary antibodies, rinsed again, incubated with Dako streptavidin-peroxidase reagent for an additional 30 minutes, and developed using a 3-amino-9-ethylcarbazole chromogen solution for 10 minutes at room temperature. The slides were subsequently counterstained with hematoxylin for 2-5 minutes.

Immunofluorescence studies. Frozen tissue sections were fixed in ice-cold acetone, blocked with normal mouse, rat, and goat serum (5\%), washed with $\times 1$ PBS, then stained with the following antibodies in the appropriate normal serum (1\%): 9G4 (rat, IgG2 $\mathrm{a}_{\mathrm{K}}$ ) or LC1 (mouse, IgG1) followed by biotinylated goat anti-rat Ig or anti-mouse Ig (BD Biosciences - Pharmingen), respectively, and developed with a third layer of SAAlexa 488. Sections were then blocked with Streptavidin/Biotin Blocking Kit (Vector Laboratories), washed, then stained with a mixture of antiIgD-PE (mouse, IA6-2; BD Biosciences - Pharmingen) and biotinylated anti-CD38 (mouse, HIT2; CALTAG Laboratories), or anti-Ki67 (B56; BD Biosciences - Pharmingen) followed by SA-7-aminomethylcoumarin. Sections were washed, mounted, and analyzed by fluorescence microscopy as previously described (5).

Intracellular calcium measurements. B cells were purified by negative selection (indirect B Cell Isolation Kit; Miltenyi Biotec), stained as described below, resuspended in HBSS containing $1 \mathrm{mM} \mathrm{Ca}$ and $\mathrm{Mg}$ and $1 \%$ FCS at a concentration of $2 \times 10^{6}$ cells $/ \mathrm{ml}$, and loaded with Indo-1 AM (Invitrogen Corp.) $\left(2 \mu \mathrm{M}\right.$ final) for 30 minutes at $37^{\circ} \mathrm{C}$ in the presence of Pluronic F127 (Molecular Probes Inc.). To identify naive $B$ cell populations of interest, the cells were stained with anti-CD38 and anti-CD27 to gate out memory and GC B cells (spleen and tonsil) or CD3/14/16/27 to gate out remaining non-B cells and memory B cells (peripheral blood) at the time of the calcium measurements. Calcium responses were measured on a BD FACS Vantage SE with UV excitation. Data was collected and displayed as the relative ratio of intensities of Indo fluorescence (Ca-bound Indo violet emission $405 \mathrm{~nm} /$ free Indo blue emission $485 \mathrm{~nm}$ ) for each cell over time and analyzed with FlowJo software version 6.2.1 (Tree Star Inc.). Samples were analyzed for a 30to 60 -second baseline in the respective gated naive $\mathrm{B}$ cell populations $\left(9 \mathrm{G}^{+}, 9 \mathrm{G}^{-}, \mathrm{LC1}^{+}\right)$at $37^{\circ} \mathrm{C}$ followed by the addition of $20 \mu \mathrm{g} / \mathrm{ml} \mathrm{F}\left(\mathrm{ab}^{\prime}\right)_{2}$ goat anti-human IgM or anti-IgD.

\section{Acknowledgments}

We are indebted to E.-H. Lee (Pulmonary Unit, University of Rochester) for invaluable help in the procurement of spleen specimens and to E.C.B Milner (Department of Medicine, University of Rochester) for helpful suggestions and critical reading of the manuscript. We are also indebted to F.K. Stevenson and K. Potter (Tenovus Research Laboratories) for the kind gift of the 9G4 hybridoma and to J. Spencer and D. Dunn-Walters (Guy's, King's and St. Thomas' Medical School) for sharing unpublished information. This work was supported in part by grants to J.H. Anolik (National Institute of Arthritis and Musculoskeletal and Skin Diseases K08AR048303 and the Lupus Foundation of America), I. Sanz (NIH National Institute of Allergy and Infectious Disease 
RO1 AI049660-01A1 and U19-Rochester Autoimmunity Center of Excellence AI56390), and G. Silverman (UCSD Rheumatic Diseases Core Center P30AR47360).

Received for publication December 13, 2004, and accepted in revised form July 26, 2005.

Address correspondence to: Iñaki Sanz, University of Rochester School of Medicine, 601 Elmwood Avenue, Box 695, Rochester,
New York 14642, USA. Phone (585) 275-2891; Fax: (585) 442-3214; E-mail: Ignacio_Sanz@urmc.rochester.edu.

Amedeo Cappione III's present address is: Guava Technologies, Hayward, California, USA.

Aimee Pugh-Bernard's present address is: Department of Immunology, National Jewish Medical and Research Center, Denver, Colorado, USA.
1. Hochberg, M.C. 1997. Updating the American College of Rheumatology revised criteria for the classification of systemic lupus erythematosus [letter]. Arthritis Rheum. 40:1725.

2. Winfield, J.B., Winchester, R.J., and Kunkel, H.G. 1975. Association of cold-reactive antilymphocyte antibodies with lymphopenia in systemic lupus erythematosus. Artbritis Rheum. 18:587-594.

3. Cappione, A.J., Pugh-Bernard, A.E., Anolik, J.H., and Sanz, I. 2004. Lupus IgG VH4.34 antibodies bind to a 220-kDa glycoform of CD45/B220 on the surface of human B lymphocytes. J. Immunol. 172:4298-4307.

4. Chan, O.T., Madaio, M.P., and Shlomchik, M.J. 1999. The central and multiple roles of B cells in lupus pathogenesis. Immunol. Rev. 169:107-121.

5. Pugh-Bernard, A.E., et al. 2001. Regulation of inherently autoreactive VH4-34 B cells in the maintenance of human B cell tolerance. J. Clin. Invest. 108:1061-1070.

6. Silberstein, L.E., et al. 1991. Variable region gene analysis of pathologic human autoantibodies to the related $\mathrm{i}$ and I red blood cell antigens. Blood. 78:2372-2386.

7. Stevenson, F.K., Smith, G.J., North, J., Hamblin, T.J., and Glennie, M.J. 1989. Identification of normal B-cell counterparts of neoplastic cells which secrete cold agglutinins of anti-I and anti-i specificity. Br. J. Haematol. 72:9-15.

8. Isenberg, D., Spellerberg, M., Williams, W., Griffiths, M., and Stevenson, F. 1993. Identification of the 9G4 idiotope in systemic lupus erythematosus. Br. J. Rheumatol. 32:876-882.

9. Isenberg, D.A., et al. 1998. Correlation of 9G4 idiotope with disease activity in patients with systemic lupus erythematosus. Ann. Rheum. Dis. 57:566-570.

10. van Vollenhoven, R.F., et al. 1999. VH4-34 encoded antibodies in systemic lupus erythematosus: a specific diagnostic marker that correlates with clinical disease characteristics. J. Rheumatol. 26:1727-1733.

11. William, J., Euler, C., Christensen, S., and Shlomchik, M.J. 2002. Evolution of autoantibody responses via somatic hypermutation outside of germinal centers. Science. 297:2066-2070.

12. Carsetti, R., Rosado, M.M., and Wardmann, H. 2004. Peripheral development of B cells in mouse and man. Immunol. Rev. 197:179-191.

13. Weller, S., et al. 2001. CD40-CD40L independent Ig gene hypermutation suggests a second B cell diversification pathway in humans. Proc. Natl. Acad. Sci. U. S. A. 98:1166-1170.

14. Zheng, N.-Y., et al. 2004. Human immunoglobulin selection associated with class switch and possible tolerogenic origins for Cdelta class-switched B cells. J. Clin. Invest. 113:1188-1201. doi:10.1172/ JCI200420255.

15. Mockridge, C.I., et al. 2004. Common patterns of $B$ cell perturbation and expanded V4-34 immunoglobulin gene usage in autoimmunity and infection. Autoimmunity. 37:9-15.

16. Dorner, T., Heimbacher, C., Farner, N.L., and Lipsky, P.E. 1999. Enhanced mutational activity of Vkappa gene rearrangements in systemic lupus erythematosus. Clin. Immunol. 92:188-196.

17. Dunn-Walters, D.K., Isaacson, P.G., and Spencer, J.
1995. Analysis of mutations in immunoglobulin heavy chain variable region genes of microdissected marginal zone (MGZ) B cells suggests that the MGZ of human spleen is a reservoir of memory B cells. J. Exp. Med. 182:559-566.

18. Lopes-Carvalho, T., and Kearney, J.F. 2004. Development and selection of marginal zone B cells. Immunol. Rev. 197:192-205.

19. Weller, S., et al. 2004. Human blood IgM "memory" $B$ cells are circulating splenic marginal zone B cells harboring a pre-diversified immunoglobulin repertoire. Blood. 104:3647-3654.

20. Suzuki, I., Pfister, L., Glas, A., Nottenburg, C., and Milner, E.C. 1995. Representation of rearranged $\mathrm{VH}$ gene segments in the human adult antibody repertoire. J. Immunol. 154:3902-3911.

21. MacLennan, I.C. 1994. Germinal centers. Annu. Rev. Immunol. 12:117-139.

22. Pascual, V., Wilson, P., Liu, Y.J., Banchereau, J., and Capra, J.D. 1997. Biased VH4 gene segment repertoire in the human tonsil. Chem. Immunol. 67:45-57.

23. Dolmetsch, R.E., Lewis, R.S., Goodnow, C.C., and Healy, J.I. 1997. Differential activation of transcription factors induced by $\mathrm{Ca} 2+$ response amplitude and duration. Nature. 386:855-858.

24. Mandik-Nayak, L., Bui, A., Noorchashm, H., Eaton, A., and Erikson, J. 1997. Regulation of anti-doublestranded DNA B cells in nonautoimmune mice: localization to the T-B interface of the splenic follicle. J. Exp. Med. 186:1257-1267.

25. Wardemann, H., et al. 2003. Predominant autoantibody production by early human B cell precursors. Science. 301:1374-1377.

26. Samuels, J., Ng, Y.-S., Coupillaud, C., Paget, D., and Meffre, E. 2005. Impaired early B cell tolerance in patients with rheumatoid arthritis. J. Exp. Med. 201:1659-1667.

27. Yurasov, S., et al. 2005. Defective B cell tolerance checkpoints in systemic lupus erythematosus. J. Exp. Med. 201:703-711.

28. Rathmell, J.C., et al. 1995. CD95 (Fas)-dependent elimination of self-reactive $\mathrm{B}$ cells upon interaction with CD4+ T cells. Nature. 376:181-184.

29. Linton, P., Rudie, A., and Klinman, N. 1991. Tolerance susceptibility of newly generating memory B cells. J. Immunol. 146:4099-4104.

30. Han, S., et al. 1995. Cellular interaction in germinal centers. Roles of CD40 ligand and B7-2 in established germinal centers. J. Immunol. 155:556-567.

31. Pulendran, B., Smith, K.G., and Nossal, G.J. 1995. Soluble antigen can impede affinity maturation and the germinal center reaction but enhance extrafollicular immunoglobulin production. J. Immunol. 155:1141-1150.

32. Shokat, K.M., and Goodnow, C.C. 1995. Antigen-induced B-cell death and elimination during germinal-centre immune responses. Nature. 375:334-338.

33. Paul, E., Lutz, J., Erikson, J., and Carroll, M.C. 2004. Germinal center checkpoints in B cell tolerance in 3H9 transgenic mice. Int. Immunol. 16:377-384.

34. Notidis, E., Heltemes, L., and Manser, T. 2002. Dominant, hierarchical induction of peripheral tolerance during foreign antigen-driven $\mathrm{B}$ cell development. Immunity. 17:317-327.

35. Cyster, J.G., Hartley, S.B., and Goodnow, C.C. 1994. Competition for follicular niches excludes self-reactive cells from the recirculating B-cell repertoire. Nature. 371:389-395.

36. Hannum, L.G., Ni, D., Haberman, A.M., Weigert, M.G., and Shlomchik, M.J. 1996. A disease-related rheumatoid factor autoantibody is not tolerized in a normal mouse: implications for the origins of autoantibodies in autoimmune disease. J. Exp. Med. 184:1269-1278.

37. Liu, X., and Manser, T. 2005. Antinuclear antigen B cells that down-regulate surface B cell receptor during development to mature, follicular phenotype do not display features of anergy in vitro. J. Immunol. 174:4505-4515.

38. Acevedo-Suarez, C.A., Hulbert, C., Woodward, E.J., and Thomas, J.W. 2005. Uncoupling of anergy from developmental arrest in anti-insulin B cells supports the development of autoimmune diabetes. J. Immunol. 174:827-833.

39. Benschop, R.J., et al. 2001. Activation and anergy in bone marrow B cells of a novel immunoglobulin transgenic mouse that is both hapten specific and autoreactive. Immunity. 14:34-43.

40. Foote, L.C., Marshak-Rothstein, A., and Rothstein, T.L. 1998. Tolerant B lymphocytes acquire resistance to Fas-mediated apoptosis after treatment with interleukin 4 but not after treatment with specific antigen unless a surface immunoglobulin threshold is exceeded. J. Exp. Med. 187:847-853.

41. Cooke, M.P., et al. 1994. Immunoglobulin signal transduction guides the specificity of B cell-T cell interactions and is blocked in tolerant self-reactive B cells. J. Exp. Med. 179:425-438.

42. Roark, J.H., Bui, A., Nguyen, K.A., Mandik, L., and Erikson, J. 1997. Persistence of functionally compromised anti-double-stranded DNA B cells in the periphery of non-autoimmune mice. Int. Immunol. 9:1615-1626.

43. Lesley, R., et al. 2004. Reduced competitiveness of autoantigen-engaged $\mathrm{B}$ cells due to increased dependence on BAFF. Immunity. 20:441-453.

44. de Vinuesa, C.G., et al. 2000. Germinal centers without T cells. J. Exp. Med. 191:485-494.

45. Vora, K.A., et al. 2003. Cutting edge: germinal centers formed in the absence of B cell-activating factor belonging to the TNF family exhibit impaired maturation and function. J. Immunol. 171:547-551.

46. Rahman, Z.S., Rao, S.P., Kalled, S.L., and Manser, T. 2003. Normal induction but attenuated progression of germinal center responses in BAFF and BAFF-R signaling-deficient mice. J. Exp. Med. 198:1157-1169.

47. Lentz, V.M., and Manser, T. 2001. Cutting edge: germinal centers can be induced in the absence of T cells. J. Immunol. 167:15-20.

48. Pillai, S., Cariappa, A., and Moran, S.T. 2005. Marginal zone B cells. Annu. Rev. Immunol. 23:161-196.

49. Grimaldi, C.M., Hicks, R., and Diamond, B. 2005. B cell selection and susceptibility to autoimmunity. J. Immunol. 174:1775-1781.

50. Li, Y., Li, H., and Weigert, M. 2002. Autoreactive B cells in the marginal zone that express dual receptors. 
J. Exp. Med. 195:181-188.

51. Rui, L., Vinuesa, C.G., Blasioli, J., and Goodnow, C.C. 2003. Resistance to CPG DNA-induced autoimmunity through tolerogenic $B$ cell antigen receptor ERK signaling. Nat. Immunol. 4:594-600.

52. Seo, S.J., et al. 2002. The impact of Thelper and $\mathrm{T}$ regulatory cells on the regulation of anti-doublestranded DNA B cells. Immunity. 16:535-546

53. Vinuesa, C.G., et al. 2005. A RING-type ubiquitin ligase family member required to repress follicular helper T cells and autoimmunity. Nature. 435:452-458.

54. Leadbetter, E.A., et al. 2002. Chromatin-IgG complexes activate B cells by dual engagement of IgM and Toll-like receptors. Nature. 416:603-607.

55. Spellerberg, M.B., Chapman, C.J., Mockridge, C.I., Isenberg, D.A., and Stevenson, F.K. 1995. Dual recognition of lipid A and DNA by human antibodies encoded by the VH4-21 gene: a possible link between infection and lupus. Hum. Antibodies Hybridomas. 6:52-56

56. Pugh-Bernard, A., Hocknell, K., Cappione, A., Anolik, J., and Sanz, I. 2002. VH4-34 anti-I/i autoantibodies recognize apoptotic cells. Arthritis Rheum. 46:S126.

57. Devitt, A., et al. 1998. Human CD14 mediates recognition and phagocytosis of apoptotic cells. Nature. 392:505-509.

58. Viglianti, G.A., et al. 2003. Activation of autoreactive B cells by CpG dsDNA. Immunity. 19:837-847.

59. Baumann, I., et al. 2002. Impaired uptake of apoptotic cells into tingible body macrophages in germinal centers of patients with systemic lupus erythematosus. Arthritis Rheum. 46:191-201.

60. Cohen, P.L., et al. 2002. Delayed apoptotic cell clearance and lupus-like autoimmunity in mice lacking the c-mer membrane tyrosine kinase. J. Exp. Med. 196:135-140.

61. Bave, U., Alm, G.V., and Ronnblom, L. 2000. The combination of apoptotic U937 cells and lupus IgG is a potent IFN-alpha inducer. J. Immunol. 165:3519-3526.

62. Thien, M., et al. 2004. Excess BAFF rescues selfreactive $B$ cells from peripheral deletion and allows them to enter forbidden follicular and marginal zone niches. Immunity. 20:785-798.

63. Gross, J.A., et al. 2000. TACI and BCMA are receptors for a TNF homologue implicated in B-cell autoimmune disease. Nature. 404:995-999.

64. Zhang, J., et al. 2001. Cutting edge: a role for B lymphocyte stimulator in systemic lupus erythematosus.
J. Immunol. 166:6-10.

65. Arnett, F.C., et al. 1988. The American Rheumatism Association 1987 revised criteria for the classification of rheumatoid arthritis. Arthritis Rheum. 31:315-324.

66. Cary, S.P., Lee, J., Wagenknecht, R., and Silverman, G.J. 2000. Characterization of superantigen-induced clonal deletion with a novel clan IIIrestricted avian monoclonal antibody: exploiting evolutionary distance to create antibodies specific for a conserved V-H region surface. J. Immunol. 164:4730-4741

67. Potter, K.N., Li, Y.C., and Capra, J.D. 1994. The cross-reactive idiotopes recognized by the monoclonal antibodies 9G4 and LC1 are located in framework region 1 of two non-overlapping subsets of human VH4 family encoded antibodies. Scand. J. Immunol. 40:43-49.

68. Odendahl, M., et al. 2000. Disturbed peripheral B lymphocyte homeostasis in systemic lupus erythematosus. J. Immunol. 165:5970-5979.

69. Carsetti, R., Kohler, G., and Lamers, M.C. 1995. Transitional B cells are the target of negative selection in the B cell compartment. J. Exp. Med. 181:2129-2140 